\title{
NONCOMMUTATIVE TOPOLOGICAL DYNAMICS. I
}

BY

DANIEL AVITZOUR

\begin{abstract}
Ergodicity and minimality are defined for $C^{*}$-flows $(G, A)$ where $G$ is a group acting on a $C^{*}$-algebra $A$ by ${ }^{*}$-automorphisms. Elementary properties are proved and several examples are given. In particular, an example shows that there are arbitrarily large $C^{*}$-algebras admitting a minimal action of the integers.
\end{abstract}

0. Introduction. The aim of this paper is to initiate a study of $C^{*}$-dynamical systems from the point of view of topological dynamics. Concepts and results usually defined for topological flows $(G, X)$, where $G$ is a group acting on a compact space $X$ by homeomorphisms, are generalized to the context of $C^{*}$-flows $(G, A)$ where $A$ is a (unital) $C^{*}$-algebra and $G$ acts on $A$ by ${ }^{*}$-automorphisms.

In this paper I shall discuss ergodicity and minimality. I prove their elementary properties and show by examples that they do not imply separability of $A$ in the case $G=Z$ in contrast to the situation in topological flows. A subsequent paper [3] will deal with weak mixing and almost periodicity.

$\S 1$ discusses some preliminary facts about semicontinuous affine functions. $\$ 2$ deals with general properties of subalgebras and inductive limits. $\S 33,4$ deal with ergodicity and minimality with some examples.

Throughout this paper " $C^{*}$-algebra" means unital $C^{*}$-algebra and "subalgebra" means sub- $C^{*}$-algebra containing the unit except when referring to hereditary subalgebras. These are not assumed to contain the unit. We shall identify the multiples of the unit with the complex numbers.

\section{Semicontinuous affine functions.}

1.0. Let $X$ be a compact Hausdorff space. Denote by $M_{1}(X)$ the set of probability measures on $X$ with the vague topology. $X$ can be embedded in $M_{1}(X)$, identifying the point $x \in X$ with the point measure $\delta_{x} \in M_{1}(X)$.

Bounded l.s.c. (lower semicontinuous) real-valued functions on $X$ can be identified with bounded affine 1.s.c. functions on $M_{1}(X)$. If $f$ is a function on $X$, the corresponding function on $M_{1}(X)$ takes $\mu \in M_{1}(X)$ to $\mu(f)$. This is an extension of $f$.

A bounded l.s.c. function on $X$ achieves its infimum on a (nonempty) closed set.

Received by the editors August 18, 1981 and, in revised form, December 22, 1982. 1980 Mathematics Subject Classification. Primary 46L55. 
If $f$ is any bounded function on $X \operatorname{let} \lim f$ be its lower envelope,

$$
(\underline{\lim } f)(x)=\underline{\lim _{y \rightarrow x}} f(y) \text {. }
$$

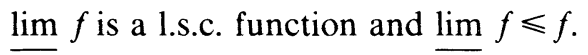

1.1. Lemma. A bounded l.s.c. function has a meager set of discontinuity points.

Proof. Recall that the oscillation of $f$, defined by

$$
(O f)(x)=\inf \{\sup \{|f(y)-f(z)|: y, z \in U\}, U \operatorname{nbd} \text { of } x\},
$$

is an upper-semicontinuous function, and $f$ is continuous at $x \in X$ iff $(O f)(x)=0$. The set of discontinuity points of $f$ is

$$
\bigcup_{n=1}^{\infty}\{x \in X:(\text { Of })(x) \geqslant 1 / n\} .
$$

I want to show that the closed set $\{x \in X:(O f)(x) \geqslant 1 / n\}$ has an empty interior. Suppose $x_{1}$ is in the interior of that set. In a small enough nbd of $x_{1}$, there is a point $x_{2}$ such that $\left|f\left(x_{2}\right)-f\left(x_{1}\right)\right| \geqslant 1 / 2 n$. If the nbd is small enough, then because $f$ is l.s.c. it is impossible that $f\left(x_{2}\right) \leqslant f\left(x_{1}\right)-1 / 2 n$, and we must have $f\left(x_{2}\right) \geqslant f\left(x_{1}\right)+$ $1 / 2 n$. Also if the nbd is small enough, $x_{2} \in\{x:(O f)(x) \geqslant 1 / n\}$. Apply the same process to $x_{2}$ to obtain a point $x_{3}$. We get a sequence $\left\{x_{m}\right\}$ such that $f\left(x_{m+1}\right) \geqslant$ $f\left(x_{m}\right)+1 / 2 n$ and this contradicts the assumption that $f$ is bounded. Q.E.D.

1.2. LEMMA. Let $f$ be a bounded l.s.c. function on $X$. $f$ is constant on a dense set in $X$ iff $f$ is constant on a dense set in $M_{1}(X)$.

Proof. If $D \subseteq X$ is dense, $\operatorname{conv}(D) \subseteq M_{1}(X)$ is dense. This proves immediately one direction.

Now assume $f=0$ on a dense set in $M_{1}(X)$. Since $f$ is 1.s.c., $f \geqslant 0$ on $M_{1}(X)$, in particular on $X$. Assume $f \geqslant 0$ on a nonempty open set in $X$. We can find a smaller nonempty open set $U$, such that $f \geqslant \varepsilon>0$ on $U$. Here we use the fact that 1.s.c. functions on compact sets achieve their minimum. The set $\left\{\mu \in M_{1}(X): \mu(U)>\frac{1}{2}\right\}$ is open, so it contains a measure $\mu_{0}$ such that $\mu_{0}(f)=0$. We get a contradiction since $\mu_{0}(f) \geqslant \varepsilon \mu_{0}(U) \geqslant \frac{1}{2} \varepsilon>0$. Q.E.D.

1.3. Let $K$ be a compact convex set in a locally convex linear topological space. Denote by $A(K)$ the continuous affine real functions on $K$ equipped with sup-norm. Now $A(K)$ is an order-unit space, and the second dual of $A(K)$ can be identified with $A^{b}(K)$, the bounded affine functions on $K$. For details see [12].

If $A$ is a $C^{*}$-algebra and $K$ its set of states, the selfadjoint elements of $A$ can be identified with $A(K)$ and we shall always assume this identification (see [12]).

The 1.s.c. functions in $A^{b}(K)$ are denoted $A^{m}(K)$, or $A^{m}$ when considered as elements of $A^{* *}$. Elements of $A^{m}$ achieve their minimum on a (nonempty) closed face of $K$.

If $f \in A^{b}(K)$ then $\lim f \in A^{m}(K)$ : we only have to show that $\lim f$ is affine. Let $\phi, \psi \in K$ and let $U, \overline{V \text { be }}$ nbds of $\phi, \psi$ respectively. Let $0<\lambda<1$.

$$
\inf \{f: \lambda U+(1-\lambda) V\}=\lambda \inf \{f: U\}+(1-\lambda) \inf \{f: V\} .
$$


Since nbds of the form $\lambda U+(1-\lambda) V$ are a base at $\lambda \phi+(1-\lambda) \psi$, we obtain

$$
(\underline{\lim } f)(\lambda \phi+(1-\phi) \psi)=\lambda(\underline{\lim f})(\phi)+(1-\lambda)(\underline{\lim f})(\psi) \text {. Q.E.D. }
$$

Let $f \in A^{b}(K)$. Then $O f \in-A^{m}(K)$. We only have to prove that $O f$ is affine. This follows easily from the definition of $O f$ once we notice that the absolute value sign in the definition can be omitted.

Now let $f: K \rightarrow R^{n}$ be affine bounded. For any $u \in R^{n}$ let $f_{u}(\phi)=\langle f(\phi), u\rangle$. Then $f_{u} \in A^{b}(K)$.

\subsection{Proposition. The function $u \rightarrow O f_{u}$ from $R^{n}$ to $-A^{m}(K)$ is continuous.}

Proof. It is easy to verify that for $\phi \in K$ fixed, the function $u \rightarrow\left(O f_{u}\right)(\phi)$ is a seminorm on $R^{n}$, the value of which is bounded by $2\|f\|$ on the unit ball.

For $u, v \in R^{n}$ we get ${ }^{\prime}\left|\left(O f_{u}\right)(\phi)-\left(O f_{v}\right)(\phi)\right| \leqslant 2\|f\|\|u-v\|$. So $\left\|O f_{u}-O f_{v}\right\| \leqslant$ $2\|f\|\|u-v\|$ Q.E.D.

DEFINITION. The average oscillation of $f$ is

$$
O_{A} f=\int_{B^{n}} O f_{u} d u \in-A^{m}(K)
$$

where integration is with respect to normalized Lebesgue measure on the unit ball (or unit sphere) in $R^{n}$.

1.5. Proposition. The average oscillation is zero exactly at the points of continuity.

Proof. If $\left(O_{A} f\right)(\phi)=0$ then $\left(O f_{u}\right)(\phi)=0$ for all $u \in B^{n}$, i.e., $f_{u}$ is continuous at $\phi$ for all $u \in B^{n}$ so $f$ is continuous at $\phi$. Q.E.D.

\section{Factors, inductive limits, opposites.}

2.0. Let $A$ be a $C^{*}$-algebra. There are 1-1 correspondences between (norm-closed) left ideals in $A$, order ideals in $A$, hereditary subalgebras of $A$, weak-* closed order ideals in $A^{*}$, weak-* closed faces of $K$ - the set of states of $A$, and open projections in $A^{* *}$. We shall use these correspondences repeatedly. For details see [12].

If $\phi$ is a state of $A$, denote by $A_{\phi}$ the hereditary subalgebra

$$
A_{\phi}=\left\{a \in A: \phi\left(a^{*} a\right)=\phi\left(a a^{*}\right)=0\right\} .
$$

Every hereditary subalgebra is an intersection of $A_{\phi}-s$ with $\phi$ pure.

2.1. Proposition. Let $A$ be a $C^{*}$-algebra and $B$ a subalgebra of $A$. The hereditary subalgebras of $B$ are $\{J \cap B: J$ hereditary subalgebra of $A\}$.

Proof. For $J \subseteq A$ hereditary, it is clear that $J \cap B$ is a hereditary subalgebra of $B$.

Let $j$ be a hereditary subalgebra of $B . j$ is the intersection of a family $\left\{B_{\phi}: \phi \in I\right\}$, where $I$ is a set of states of $B$. Extend each $\phi \in I$ to a state $\tilde{\phi}$ of $A$, and let $J=\cap\{A \tilde{\phi}: \phi \in I\}$, a hereditary subalgebra of $A$. Now $j=J \cap B$. Q.E.D. 
2.2. Proposition. Let $A$ be $a C^{*}$-algebra and $\left\{A_{n}\right\}$ an increasing sequence of subalgebras, such that $A=\left(\cup A_{n}\right)^{-}$.

The map $J \rightarrow\left\{J \cap A_{n}\right\}_{n=1}^{\infty}$ is a 1-1 correspondence between hereditary subalgebras of $A$, and sequences of hereditary subalgebras $\left\{J_{n}\right\}, J_{n} \subseteq A_{n}$, such that $J_{n-1}=J_{n} \cap$ $A_{n-1}$.

Proof. The map is well defined since $J \cap A_{n-1}=\left(J \cap A_{n}\right) \cap A_{n-1}$. We have to show that, given $\left\{J_{n}\right\}$ as above, there is a unique hereditary subalgebra $J$ of $A$, such that $J \cap A_{n}=J$. Using the correspondence between hereditary subalgebras and order ideals in the dual space, what we have to prove is: given a sequence $\left\{R_{n}\right\}$ of weak-* closed order ideals in $A_{n}^{*}$ such that $R_{n \mid A_{n-1}}=R_{n-1}$, there is a unique order ideal $R$ in $A^{*}$, such that $R_{\mid A_{n}}=R_{n}$.

Existence. Let $R=\left\{f \in\left(A^{*}\right)^{+}: f_{\left.\right|_{A_{n}}} \in R_{n}\right.$ all $\left.n\right\} . R$ is a closed order ideal, and $R_{\mathrm{LA}_{n}} \subseteq R_{n}$. For the reverse inclusion, let $f \in R_{n}$. For each $m \geqslant n$ extend $f$ to some $f_{m} \in\left(A_{m}^{*}\right)^{+}$, then extend $f_{m}$ to some $\tilde{f}_{m} \in\left(A^{*}\right)^{+}$. Now $\left\|\tilde{f}_{m}\right\|=\tilde{f}_{m}(1)=f(1)$ so $\left\{\tilde{f}_{m}\right\}$ has an accumulation point $\tilde{f}$ in $\left(A^{*}\right)^{+}$. For all $m, \tilde{f}_{\left.\right|_{m}} \in R_{m}$, so $\tilde{f} \in R$. Finally $f=\tilde{f}_{\mathcal{A}_{n}} \in R_{\mathrm{HA}_{n}}$.

Uniqueness. Suppose $R_{\mathbf{A A}_{n}}=R_{\mathbf{A A}_{n}}^{\prime}$ for all $n$. Let $f \in R$. For each $n$ there is $f_{n} \in R^{\prime}$ such that $f=f_{n}$ on $A_{n} \cdot\left\|f_{n}\right\|=f_{n}(1)=f(1)$, so $\left\{f_{n}\right\}$ has an accumulation point $f^{\prime}$ in $R^{\prime}$. Since $f=f^{\prime}$ on $A_{n}$ for each $n$, and $\cup\left\{A_{n}\right\}$ is dense, $f=f^{\prime} \in R^{\prime}$ so $R \subseteq R^{\prime}$.

The proof uses the correspondence between hereditary subalgebras and order ideals in the dual, in an essential way. Hereditary subalgebras were used to make the map well defined, and order ideals to show that it is 1-1 and onto. Q.E.D.

Corollary. Let $A, A_{n}$ be as in the proposition. Let $P$ be a positive map defined on A. $P$ is faithful on $A$ iff it is faithful on $A_{n}$ for each $n$.

Proof. Let $A_{P}=\left\{a \in A: P\left(a^{*} a\right)=P\left(a a^{*}\right)=0\right\} . P$ is faithful iff $A_{P}=0 . P$ is faithful on $A_{n}$ iff $A_{P} \cap A_{n}=0$. Q.E.D.

$C^{*}$-flows are the main objects of interest in this work. A $C^{*}$-flow $(G, A), G$ a topological group, $A$ a $C^{*}$-algebra, is the same as a $C^{*}$-dynamical system, as defined in [12] or other texts: $G$ acts on $A$ via a continuous homomorphism into the automorphism group of $A$. If $B \subseteq A$ is a $G$-invariant subalgebra, we call the $C^{*}$-flow $(G, B)$, a factor of $(G, A)$ as customary in topological dynamics.

2.3. Proposition. Let $(G, A)$ be a $C^{*}$-flow and let $(G, B)$ be a factor of $(G, A)$. The $G$-invariant, hereditary subalgebras of $B$ are $\{J \cap B: J$ a G-invariant, hereditary subalgebra of $A$ \}.

Proof. Let $j \subseteq B$ be $G$-invariant hereditary. $j$ is the intersection of a family $\left\{B_{\phi}\right.$ : $\phi \in I\}, I$ a set of states of $B$. But since $j$ is $G$-invariant, it is also the intersection of: $\left\{B_{s \phi}: \phi \in I, s \in G\right\}$. Extend each $\phi \in I$ to a state $\tilde{\phi}$ on $A$. Let

$$
J=\cap\left\{A_{s \tilde{\phi}}: \phi \in I, s \in G\right\} .
$$

Now $J$ is $G$-invariant and $j=J \cap B$. Q.E.D.

2.4. Proposition. Let $(G, A)$ be a $C^{*}$-flow. Let $\left(G, A_{n}\right)$ be increasing factors of $(G, A)$, such that $A=\left(\cup A_{n}\right)^{-}$. 
The map $J \rightarrow\left\{J \cap A_{n}\right\}$ is a 1-1 correspondence between G-invariant hereditary subalgebras of $A$, and sequences $\left\{J_{n}\right\}$ of $G$-invariant, hereditary subalgebras of $A_{n}$, such that $J_{n-1}=J_{n} \cap A_{n-1}$.

Proof. Follows immediately from 2.2. Q.E.D.

2.5. Let $A$ be a $C^{*}$-algebra. The opposite algebra, denoted $A^{\text {op }}$, has the same elements, linear operations, and *-operation as $A$, and multiplication defined by $a \otimes b=b a$.

$A$ and $A^{\text {op }}$, share the same order structure. They have the same positive elements, states, hereditary subalgebras and automorphisms. If $(G, A)$ is a $C^{*}$-flow, we have a naturally defined opposite flow $\left(G, A^{\mathrm{op}}\right)$.

\section{Ergodicity.}

3.0. Recall that a transformation group $(G, X)$ is called ergodic if there is a dense $G$-orbit in $X$, or if every $G$-invariant nonempty, open subset of $X$ is dense [7].

Lemma. A transformation group $(G, X)$ is ergodic iff every $G$-invariant, bounded, l.s.c. function on $X$ is constant on a dense subset.

Proof. The condition is sufficient since, if $U \subseteq X$ is open and $G$-invariant, then $1_{U}$ is a $G$-invariant, bounded, l.s.c. function.

To show that it is necessary, let $f$ be a $G$-invariant, bounded, 1.s.c. function on $X$. Choose $x \in X$ with a dense orbit. Given $y \in X$, there is a net $\left\{s_{\alpha}\right\}$ in $G$, such that $s_{\alpha} x \rightarrow y$. By l.s.c. and $G$-invariance of $f, f(x) \geqslant f(y)$. Hence $f$ is equal to the constant $\sup (f)$ on the dense subset $\left\{x:(G x)^{-}=X\right\}$. Q.E.D.

Corollary. $(G, X)$ is ergodic iff every $G$-invariant, bounded, l.s.c., affine function on $M_{1}(X)$ is constant on a dense subset of $M_{1}(X)$.

Proof. Use 1.2. Q.E.D.

3.1. Proposition. Let $(G, A)$ be a $C^{*}$-flow. Let $K$ be the set of states of $A$. The following are equivalent:

(1) $a \in\left(A^{m}\right)_{G} \Rightarrow a$ is constant on a comeager subset of $K$.

(2) $a \in\left(A^{m}\right)_{G} \Rightarrow a$ is constant on a dense subset of $K$.

(3) $a \in\left(A^{m}\right)_{G} \Rightarrow a$ is constant on its set of continuity points, $C(a)$.

(4) $a \in\left(A^{b}\right)_{G}, C(a)$ comeager $\Rightarrow a$ is constant on a comeager subset of $K$.

(5) $a \in\left(A^{b}\right)_{G}, C(a)$ comeager $\Rightarrow a$ is constant on a dense subset of $K$.

(6) $a \in\left(A^{b}\right)_{G}, C(a)$ comeager $\Rightarrow a$ is constant on $C(a)$.

Proof. $(1) \Rightarrow(2),(4) \Rightarrow(5) . K$ is a Baire space.

$(2) \Rightarrow(3),(5) \Rightarrow(6)$. If a function is constant on a dense set, it is equal to that constant at every point of continuity.

(3) $\Rightarrow(1),(6) \Rightarrow(4) . C(a)$ is comeager.

(4) $\Rightarrow$ (1). If $a \in A^{m}, a$ is comeager.

(1) $\Rightarrow(4)$. Let $a \in\left(A^{b}\right)_{G}$. Since $\lim a \in\left(A^{m}\right)_{G}$ (see 1.3), $\lim a$ is constant on a comeager set $D$. On $C(a), a=\lim \bar{a}$, so $a$ is constant on the comeager set $D \cap C(a)$. Q.E.D. 
3.2. Definition. A $C^{*}$-flow $(G, A)$ is ergodic if it satisfies (1)-(6) of 3.1. By 3.0 the definition of ergodicity reduces to the usual one in the commutative case.

\subsection{Proposition. Ergodicity passes to factors and opposites.}

Proof. Let $(G, A)$ be an ergodic flow, and $(G, B)$ a factor. Let $\pi: K_{A} \rightarrow K_{B}$ be the restriction map, which is a continuous surjection.

If $a \in\left(B^{m}\right)_{G}$, then $a \pi \in\left(A^{m}\right)_{G}$ so $a \pi$ is constant on a dense set $D \subseteq K_{A}$. So $a$ is constant on the dense subset $\pi(D) \subseteq K_{B}$. We get $(G, B)$ is ergodic.

Since $\left(A^{\mathrm{op}}\right)^{m}=A^{m}$, ergodicity passes to the opposite flow. Q.E.D.

3.4. Proposition. Let $(G, A)$ be a $C^{*}$-flow. Let $(u, \pi)$ be a covariant representation of $(G, A)$, such that $\pi$ is faithful. If the $W^{*}$-flow $\left(G, \pi(A)^{\prime \prime}\right)$ is ergodic, then $(G, A)$ is ergodic.

Proof. Recall that a $W^{*}$-flow $(G, M)$ is ergodic if $M_{G}=\mathbf{C}$ (see e.g. [8]). Let $a \in\left(A^{* *}\right)_{G}$. Now $\pi(a) \in\left(\pi(A)^{\prime \prime}\right)_{G}=\mathbf{C}$ so let $\lambda \in \mathbf{C}$ such that $\pi(a-\lambda)=0$. If $\xi$ is a vector in the space carrying $\pi$, and $\phi$ is the corresponding vector state, then

$$
|\phi(a-\lambda)| \leqslant \phi((a-\lambda) *(a-\lambda))^{1 / 2}=\|\pi(a-\lambda) \xi\|=0 .
$$

Since $\pi$ is faithful, combinations of vector states are dense in $K$, so $a$ is equal to $\lambda$ on a dense subset of $K$. Q.E.D.

3.5. Proposition. Let $(G, A)$ be a $C^{*}$-flow, and $H$ a dense subgroup of $G .(G, A)$ is ergodic iff $(H, A)$ is ergodic.

Proof. It suffices to show that $\left(A^{m}\right)_{H}=\left(A^{m}\right)_{G}$, and since $H \subseteq G$, the nontrivial part is $\left(A^{m}\right)_{H} \subseteq\left(A^{m}\right)_{G}$. Let $a \in\left(A^{m}\right)_{H}$ and $\phi \in K$. For all $h \in H$ we have $a(h \phi)=$ $a(\phi)$. Since $H$ is dense in $G$, and $a$ is l.s.c., $a(s \phi) \geqslant a(\phi)$ for all $s \in G$. This proves $a \in\left(A^{m}\right)_{G}$ for all $\phi \in K$.

3.6. A projection $p \in A^{* *}$ is called dense if the only closed projection greater than $p$ is 1 .

Lemma. A projection $p \in A^{* *}$ is dense iff $\{\phi \in K: \phi(p)=1\}$ is dense in $K=K_{A}$.

Proof. (1) Suppose that $\{\phi \in K: \phi(p)=1\}$ is dense and let $q \geqslant p$ be closed. $q \geqslant 1$ on the dense set $\{\phi \in K: \phi(p)=1\}$, and since $q$ is upper semicontinuous on $K, q=1$ on all of $K$.

(2) Let $F=\{\phi \in K: \phi(p)=1\}^{-} . F$ is a closed face of $K$. To see that, let $\psi_{1}$, $\psi_{2} \in K$, and let $0<\lambda<1$, such that $\lambda \psi_{1}+(1-\lambda) \psi_{2} \in F$. Let $U_{1}, U_{2}$ be nbds of $\psi_{1}, \psi_{2}$ respectively. $\lambda U_{1}+(1-\lambda) U_{2}$ is a nbd of $\lambda \psi_{1}+(1-\lambda) \psi_{2}$, so it intersects $\{\phi \in K: \phi(p)=1\}$. Let $u_{i} \in U_{i}$ such that $\left(\lambda u_{1}+(1-\lambda) u_{2}\right)(p)=1$. This implies $u_{1}(p)=1, u_{2}(p)=1$ and since $U_{1}, U_{2}$ are arbitrary we get $\psi_{1}, \psi_{2} \in \dot{F}$. So $F$ is a closed face.

By the correspondence between closed faces and open projections, we get $F=K$ iff $\inf \{q$ closed: $q=1$ on $F\}=1$. So assume $p$ is dense and let $q$ be closed such that $q=1$ on $F$. We have to show $q=1$, and it suffices to show that $q \geqslant p$.

Since $q=1$ on $F$, for all $\phi \in K$,

$$
\phi(1-p)=0 \Rightarrow \phi(1-q)=0 .
$$


That is $\left((1-p) A^{* *}\right)^{\perp} \subseteq\left((1-q) A^{* *}\right)^{\perp}$. Here for $L$ a left ideal in $A^{* *}, L^{\perp}$ is the corresponding order ideal in $A^{*}, L^{\perp}=\{\phi: \phi(L)=0\}$ (see [5]). Hence $(1-p) A^{* *} \subseteq$ $(1-q) A^{* *}$ which means that $q \geqslant p$. Q.E.D.

By 3.1 and 3.6 ergodicity implies that the only invariant dense open projection is 1 .

3.7. Now we shall discuss an example of a $C^{*}$-flow which will be dealt with further in the sequel as we introduce more properties of $C^{*}$-flows.

Let $H$ be a Hilbert space. Denote by $K(H)$ and $B(H)$ the compact and bounded operators on $H$, respectively. Let $\tilde{K}(H) \subseteq B(H)$ be the smallest unital $C^{*}$-algebra containing $K(H)$. The double dual and the multiplier algebra of $K(H)$ are the same, $B(H)$. Thus, $K(H)$ can be seen as a noncommutative analog of a discete topological space, and $\tilde{K}(H)$ as a noncommutative analog of the one-point compactification of a discrete space.

The hereditary subalgebra of $\tilde{K}(H)$ corresponding to the open projection $p \in$ $B(H)$, is

$$
K(V)=\left\{T \in K(H): T V \subseteq V \text { and } T V^{\perp}=0\right\}
$$

where $V=p H$. In $\tilde{K}(H)$ we have, in addition to these hereditary subalgebras

$$
\tilde{K}(V)=\{T+\lambda p: T \in K(V) \text { and } \lambda \in \mathbf{C}\}
$$

for $V=p H$ finite-codimensional. It is clear from the lattice structure of the hereditary subalgebras that the only nontrivial dense open projection is the one corresponding to the hereditary subalgebra $K(H) \subseteq \tilde{K}(H)$.

The automorphisms of $\tilde{K}(H)$ are conjugations by unitary operators in $B(H)$. $C^{*}$-flows $(G, \tilde{K}(H))$ are in 1-1 correspondence with projective unitary representations of $G$ on $H$, that is, continuous homomorphisms of $G$ into the unitary group of $H$ divided by its center.

3.8. Proposition. Let $\pi$ be a projective unitary representation of $G$ on $H$. Let $(G, \tilde{K}(H))$ be the corresponding $C^{*}$-flow.

The $C^{*}$-flow is ergodic iff $\pi$ is irreducible.

Proof. If $(G, \tilde{K}(H))$ is ergodic, then every nonzero, $G$-invariant, open projection is dense. So the only nontrivial, $G$-invariant hereditary subalgebra of $\tilde{K}(H)$ is $K(H)$. But if $V \subseteq H$ is a $\pi$-invariant subspace then $K(V)$ is a $G$-invariant hereditary subalgebra. So $\pi$ is irreducible.

If $\pi$ is irreducible, $(B(H))_{G}=\mathbf{C}$ so

$$
\left((\tilde{K}(H))^{m}\right)_{G} \subseteq\left((\tilde{K}(H))^{* *}\right)_{G}=(B(H) \oplus \mathbf{C})_{G}=\mathbf{C} .
$$

We identify $(\tilde{K}(H))^{* *}$ with $B(H) \oplus \mathbf{C}$ through the inclusion $T+\lambda I \rightarrow T \oplus \lambda$, $T \in K(H), \lambda \in$ C. Q.E.D.

More examples of ergodic $C^{*}$-flows with the stronger property of "weak mixing" are discussed in [3].

\section{Minimality.}

4.0. Definition. A $C^{*}$-flow $(G, A)$ is minimal if the only $G$-invariant, open projections are 0 and 1 . Equivalently if the only $G$-invariant hereditary subalgebras of $A$ are 0 and $A$. 
4.1. Proposition. Let $(G, A)$ be a $C^{*}$-flow. The following are equivalent:

(1) $a \in\left(A^{b}\right)_{G}, C(a)$ nonempty $\Rightarrow a$ is constant.

(2) $a \in\left(A^{m}\right)_{G} \Rightarrow$ a is constant.

(3) $(G, A)$ is minimal.

Proof. (1) $\Rightarrow(2) \Rightarrow(3)$. Immediate.

$(2) \Rightarrow(1)$. Let $a \in\left(A^{b}\right)_{G}, C(a)$ nonempty. Since $\lim a$ and $\varlimsup \lim a$ are in $\left(A^{m}\right)_{G}$ and $-\left(A^{m}\right)_{G}$ respectively, they are constants. These constants are equal on $C(a)$, and since $C(a)$ is nonempty they are equal; hence $a$ is constant everywhere.

(3) $\Rightarrow(2)$. Let $a \in\left(A^{m}\right)_{G}$ and let $F$ be the (nonempty) closed face on which $a$ achieves its minimum. $F$ is $G$-invariant so by minimality $F=K$ and $a$ is constant. Here we use the correspondence between closed faces and open projections. Q.E.D.

4.2. Proposition. Minimality passes to factors, opposites and inductive limits.

Proof. The proof is immediate, since we prepared the ground in 2.4 (inductive limits) and 2.3 (factors). For opposite flows it is enough to note that $\left(A^{\text {op }}\right)^{m}=A^{m}$. Q.E.D.

4.3. Proposition. If $(G, A)$ is a minimal $C^{*}$-flow, then every $G$-invariant state on $A$ is faithful. If $G$ is amenable, the converse is also true.

Proof. Let $\phi$ be a $G$-invariant state on $A . A_{\phi}$ is a $G$-invariant, proper $\left(1 \notin A_{\phi}\right)$, hereditary subalgebra of $A$. By minimality $A_{\phi}=0$, so $\phi$ is faithful.

Now assume that $G$ is amenable, and that all $G$-invariant states are faithful. Let $J$ be a $G$-invariant, proper, hereditary subalgebra, and let $F$ be the corresponding nonempty, closed face in $K, F=\{\phi \in K: \phi(J)=0\}$. Since $F$ is a compact convex set and $G$ is an amenable group acting on $F$ by affine homeomorphisms, $F$ contains a fixed point $\phi . \phi$ is a $G$-invariant state, hence faithful. But $\phi(J)=0$ so $J=0$. Q.E.D.

4.4. Proposition. Let $(G, A)$ be a $C^{*}$-flow and let $h$ be a dense subgroup of $G$. $(G, A)$ is minimal iff $(H, A)$ is minimal.

Proof. Same as 3.5. Q.E.D.

4.5. Definition. A $C^{*}$-flow $(G, A)$ is uniquely ergodic if $A$ has exactly one $G$-invariant state. If, in addition, the $G$-invariant state is faithful, the flow is strictly ergodic.

With this definition, Proposition 4.3 implies: for $G$ amenable, strict ergodicity $\Rightarrow$ minimality.

4.6. Proposition. If $G$ is amenable, factors of uniquely (strictly) ergodic G-flows are uniquely (strictly) ergodic.

Proof. Let $(G, A)$ be uniquely ergodic and let $(G, B)$ be a factor of $(G, A)$. $B$ has a $G$-invariant state $\phi$. Extend $\phi$ to a state $\psi$ of $A$. Let $M$ be a left-invariant mean on $G$, and let $\tilde{\psi}=M\{s \psi: s \in G\}$. Clearly, $\tilde{\psi}$ is a $G$-invariant state of $A$, so uniquely determined. Since $\phi=\tilde{\psi}_{\mid B}, \phi$ is uniquely determined.

The claim about strict ergodicity follows from the fact that a faithful state is faithful on every subalgebra. Q.E.D. 
4.7. Theorem. Let $(G, A)$ be a minimal $C^{*}$-flow, and let $a \in A^{* *}$ have a finitedimensional $G$-orbit. If a has a comeager set of continuity points in $K$, then $a \in A$.

Proof. Let $D$ be the (finite-dimensional) span of $G a$. Since the action of $G$ on $D$ preserves the norm, the range of $G$ in $B(D)$ is relatively compact. We conclude that $D$ has a $G$-invariant inner product. Let $\left\{a_{1}, \ldots, a_{n}\right\}$ be an orthonormal basis for $D$ with respect to such an inner product. Let $V(s)$ be the unitary $n \times n$ matrix of $s \in G$ with respect to the basis. Let $f: K \rightarrow \mathbf{C}^{n}$ be the affine bounded function

$$
f(\phi)=\left(a_{1}(\phi), \ldots, a_{n}(\phi)\right) .
$$

We have $f(s \phi)=V(s) f(\phi)$ for all $s \in G, \phi \in K$. Each $a_{i}$ has a comeager set of continuity points, so the same is true for $f$. On the other hand, if $f$ is continuous, so are $a_{i}$, and so is $a$, which is spanned by $a_{i}$. So our theorem will be proved if we prove the following

4.8. Lemma. Let $(G, A)$ be a minimal $C^{*}$-flow, $f: K \rightarrow \mathbf{C}^{n}$ a bounded affine function and $s \rightarrow V(s)$ an $n$-dimensional unitary representation of $G$, such that $f(s \phi)=$ $V(s) f(\phi), s \in G, \phi \in K$. Then $f$ is either continuous or everywhere discontinuous.

Proof. Identifying $C^{n}$ with $R^{2 n}$ in the usual way, every unitary matrix becomes orthogonal. So it is enough to prove the lemma for a function which takes values in $R^{n}$, and assume that $V(s)$ is orthogonal.

I claim: the average oscillation $O_{A} f$ is $G$-invariant. It is easily seen that

$$
\left(O f_{u}\right)(s \phi)=O f_{V(s)^{-1} u}(\phi) \text {. }
$$

So

$$
\begin{aligned}
\left(O_{A} f\right)(s \phi) & =\int_{B^{n}}\left(O f_{u}\right)(s \phi) d u \\
& =\int_{B^{n}} O f_{V(s)^{-1} u}(\phi) d u=O_{A} f(\phi) .
\end{aligned}
$$

Since the flow is minimal, $O_{A} f$, which is a $G$-invariant u.s.c. affine function, must be a constant. If the constant is zero, $f$ is continuous. If nonzero, $f$ is everywhere discontinuous. Q.E.D.

4.9. We shall now examine several examples of minimal $C^{*}$-flows.

Proposition. Let $G, H, \pi$ be as in 3.7. The flow $(G, \tilde{K}(H))$ is minimal iff $\pi$ is irreducible and finite-dimensional.

Proof. If $(G, \tilde{K}(H))$ is minimal, then by $3.8 \pi$ is irreducible. $K(H)$ is a $G$-invariant hereditary subalgebra, so $K(H)=\tilde{K}(H)$ which means that $H$ is finitedimensional.

If $H$ is finite-dimensional, the $G$-invariant hereditary subalgebras of $K(H)$ are $K(V)$ for $V \pi$-invariant. If $\pi$ is irreducible we get minimality. Q.E.D.

Let $(G, A)$ and $(H, B)$ be $C^{*}$-flows and let an action of $H$ on $A$, commuting with $G$, be given. $H$ acts on $A \otimes_{*} B$. Let $A \otimes_{H} B$ be the fixed point algebra of that action, $A \otimes_{H} B=\left(A \otimes_{*} B\right)_{H} . G$ acts on $A \otimes_{*} B$ by $s(a \otimes b)=s a \otimes b . A \otimes_{H} B$ is $G$ invariant under this action. We obtain a twisted product flow $\left(G, A \otimes_{H} B\right)$. When $A$, 
$B$ are commutative it is easily seen that if $(G, A)$ and $(H, B)$ are minimal, so is the twisted product $\left(G, A \otimes_{H} B\right)$.

4.10. Proposition. With notations as above, if $A$ is commutative and $(G, A)$ and $(H, B)$ are minimal, then $\left(G, A \otimes_{H} B\right)$ is minimal.

Proof. Let $A=C(X)$. Let $J \subseteq A \otimes_{H} B$ be a $G$-invariant, proper, hereditary subalgebra. By $2.1 J$ is contained in a proper hereditary subalgebra of $A \otimes_{*} B$, so in a maximal one, which is of the form $\left\{f \in C(X, B): f\left(x_{0}\right) \in J\right\}$, where $x_{0} \in X$ and $j \subseteq B$ is a maximal proper subalgebra. Note that by $G$-invariance of $J$,

$$
f \in J \Rightarrow f\left(s x_{0}\right) \in J \text { for all } s \in G .
$$

Fix $s \in G, h \in H$ and let $\left\{s_{\alpha}\right\}$ be a net in $G$ such that $s_{\alpha} x_{0} \rightarrow s h x_{0}$. For $f \in J$ we have $f\left(s_{\alpha} x_{0}\right) \in j$ so $f\left(s h x_{0}\right) \in j$. Since $f \in C(X) \otimes_{H} B$, we have $f\left(s x_{0}\right) \in h j$. Varying $h$ over $H$ get

$$
f\left(s x_{0}\right) \in \cap\{h j: h \in H\}=0,
$$

using minimality of $(H, B)$. Now using minimality of $(G, X)$, we get $f=0$, so $J=0$. Q.E.D.

4.11. Let us look for examples of noncommutative $C^{*}$-algebras admitting a minimal automorphism. Matrix algebras $M_{n}$ are clearly inappropriate by $\$ 4.9$ since irreducible representations of Abelian groups are one-dimensional. Similarly it can be shown that finite dimensional algebras do not give examples of the kind we need. The next step is to look at $C(X) \otimes M_{n}$. An automorphism of this algebra is of the form $f(x) \rightarrow \alpha_{x}(f(\tau x))$, where $\tau$ is a homeomorphism of $X$ and $\alpha: X \rightarrow \operatorname{Aut}\left(M_{n}\right)$ is continuous. Using $\$ 4.10$ we can construct minimal automorphisms of this form.

Let $K$ be any finite group. Veech [13] constructed a minimal homeomorphism $(\tau, W)$ and a continuous map $h: W \rightarrow K$, such that the extension flow $(Z, W \times K)$ defined by $(w, k) \rightarrow(\tau w, h(w) \cdot k)$, is minimal.

Now let $\pi$ be an $n$-dimensional irreducible representation of $K$. It gives a minimal $C^{*}$-flow $\left(K, M_{n}\right)$. Let $K$ act on $W \times K$ by right multiplication on $K$. This action commutes with the action of $Z$ on $W \times K$ defined above. We obtain a minimal twisted product $\left(Z, C(W \times K) \otimes_{K} M_{n}\right)$. Now $C(W \times K) \otimes_{K} M_{n}$ can be identified with $C(W) \otimes M_{n}$, and we get a flow of the required form with $\alpha: W \rightarrow \operatorname{Aut}\left(M_{n}\right)$ defined by

$$
\alpha_{w}(T)=\pi(h(w)) T \pi(h(w))^{-1} .
$$

4.12. Let $G$ be a discrete group and let $G_{j}=G$ for all $j \in Z$. The identity isomorphisms $G_{j} \rightarrow G_{j+1}$ extend to an automorphism of the free product group $F=*\left\{G_{j}: j \in Z\right\}$. This extends to a ${ }^{*}$-automorphism of the reduced group $C^{*}$-algebra $C_{r}^{*}(F)$, which preserves the standard faithful trace $\phi(\phi(s)=0$ for all $s \neq e$ in $F)$.

The flow $\left(Z, C_{r}^{*}(F)\right)$ is a shift automorphism on a "small $C^{*}$-free product" as defined in [2]. By $\$ 4.1$ there the flow is uniquely ergodic. By $\$ 4.3$ above the flow is minimal. The same considerations apply to any "shift automorphism" when the group is amenable and the free product is with respect to faithful states. 
Since we may take a group $G$ as large as we like, it follows that there are arbitrarily large $C^{*}$-algebras admitting minimal actions of the integers $Z$. In particular there is no "universal" minimal flow.

This example is discussed further in [3].

5. Notes. (1) The commutative cases of most claims in this paper are folk-theorems of topological dynamics. Proposition 3.1 is an extension of [9, Theorem 3.1]. Proposition 4.1 is an extension of [10, Theorem 4].

(2) In [11] minimal $C^{*}$-flows were given a definition equivalent to ours, using left ideals instead of hereditary subalgebras. Proposition 4.3 is given an erroneous proof and then used to prove that minimality passes to factors in the amenable case. It was claimed, without a proof, that minimality passes to inductive limits. The rest of the material in this paper is new.

(3) The definitions in this paper use only the order structure of the algebra. Analogous definitions make sense for a system $(G, A)$ where $A$ is any order-unit space, and $G$ acts on $A$ by order-unit automorphisms. The results of $\$ 4.7$ remain valid in this, more general, setting. For the results of the rest of the section we have to impose some conditions on $A$, for example, that $K$, the set of states, is "strongly spectral" as defined in [1].

\section{REFERENCES}

1. E. M. Alfsen and F. W. Schultz, Noncommutative spectral theory for affine function spaces on convex sets, Mem. Amer. Math. Soc. No. 172 (1976).

2. D. Avitzour, Free products of $C^{*}$-algebras, Trans. Amer. Math. Soc. 271 (1982), 423-435.

3. __ Noncommutative topological dynamics. II, Trans. Amer. Math. Soc. 282(1984), 121-135.

4. J. Dixmier, Les $C^{*}$-algèbres et leurs représentations, Gauthier-Villars, Paris, 1964.

5. E. G. Effros, Order ideals in a $C^{*}$-algebra and its dual, Duke. Math. J. 30 (1963), 391-412.

6. H. Furstenberg, The structure of distal flows, Amer. J. Math. 85 (1963), 477-515.

7. Disjointness in ergodic theory minimal sets and a problem in Diophantine approximation, Math. Systems Theory. 1 (1967), 1-49.

8. A. Guichardet, Systemes dynamiques non commutatifs, Soc. Math. France, Paris, 1974.

9. H. B. Keynes and J. B. Robertson, On ergodicity and mixing in topological transformation groups, Duke. Math. J. 35 (1968), 809-819.

10. Eigenvalue theorems in topological transformation groups, Trans. Amer. Math. Soc. 139 (1969), 359-369.

11. D. Laison and G. Laison, Topological dynamics on $C^{*}$-algebras, Trans. Amer. Math. Soc. 204, (1975) 197-205.

12. G. K. Pedersen, $C^{*}$-algebras and their automorphism groups, London Math. Soc. Monographs, no. 14, Academic Press, London and New York, 1979.

13. W. A. Veech, Finite group extension of irrational rotations, Israel J. Math. 21 (1975), 240-259.

Department of Mathematics, University of California, Berkeley, California 94720

Current address: Elta Electronics Industries, P.O. Box 330, Ashdod, Israel 